\title{
Nitrogen NMR shieldings of 2-amino-5-nitro-6-methylpyridines
}

\author{
B. Palasek and A. Puszko \\ Department of Chemistry, Academy of Economics, 53-345 Wroclaw, Poland \\ Z. Biedrzycka, W. Sicinska and M. Witanowski \\ Institute of Organic Chemistry, Polish Academy of Sciences, 01-224 Warsaw, Poland
}

Received February 1997

Revised March 1997

\begin{abstract}
Nitrogen NMR shieldings (chemical shifts) of 2-amino-5-nitro-6-methylpyridine derivatives are assessed from the point of view of substituent-induced effects under conditions where alkyl, aryl, nitro, and nitroso moieties are substituents at the amino nitrogen. The nitro nitrogen shielding reveals only little variation upon varying the substituents, and this seems to indicate that steric hindrance which is likely to force the nitro group out of the plane of the aromatic ring reduces the $\pi$-electron conjugation with the latter, and with the amino group as well. On the other side, the pyridine nitrogen shielding shows large effects of substituents at the amino moiety, which suggests a significant conjugation between the ring and the amino group. The latter effects produce a remarkable deshielding of the pyridine nitrogen in the case of nitro and nitroso substituents at the amino group.
\end{abstract}

\section{Introduction}

Nitro derivatives of pyridine draw attention as potential fungicides [1]. It has long been known that nitrogen NMR shieldings (chemical shifts) provide an attractive means of insight into interactions between substituents and the pyridine ring from the point of view of electron charge distribution and modifications thereof in such molecular systems [2]. The compounds studied in the present work (Fig. 1) constitute an interesting example where the pyridine moiety is substituted with an electron acceptor, the nitro group, and with an electron donor, the amino group. Concurrently, the nitro group is likely to be forced out of the plane of the ring owing to the steric hindrance exerted by the methyl substituent. Thus, the lone-pair electrons at the amino moiety are likely to be involved in $\pi$-electron conjugation with the aromatic ring, while the nitro group should act as an electron acceptor via the so-called inductive effect. The molecules studied, derivatives of 2-amino-5-nitro-6-methylpyridine, are examined here from the point of view of substitution at the amino moiety, where the substituents include alkyl and aryl groups as well as $\mathrm{N}$-nitroso and $\mathrm{N}$-nitro groups. In spite of a large body of data available on nitrogen NMR of substituted pyridines [2], there has so far been no information on how such a substitution affects the electron charge distribution concerned and whether nitrogen NMR can reflect such perturbations.

Attention is drawn to the sign convention employed in the present work, as was done before [2], where a plus sign corresponds to an increase in nuclear magnetic shielding. Thus we use the term 
<smiles>[R]c1ccc([N+](=O)[O-])c(C)n1</smiles>

\begin{tabular}{|c|c|c|c|}
\hline No. & $\mathrm{R}$ & No. & $\mathrm{R}$ \\
\hline 1 & $\mathrm{NH}_{2}$ & 8 & \\
\hline 2 & $\mathrm{NHMe}$ & & \\
\hline 3 & $\mathrm{NMe}_{2}$ & 9 & \\
\hline 4 & NHEt & & | \\
\hline 5 & NHPr & 10 & $\mathrm{~N}(\mathrm{Me}) \mathrm{NO}$ \\
\hline 6 & $\mathrm{NHBu}$ & 11 & $\mathrm{~N}(\mathrm{Me}) \mathrm{NO}_{2}$ \\
\hline 7 & NHPh & 12 & $\mathrm{~N}(\mathrm{Et}) \mathrm{NO}_{2}$ \\
\hline
\end{tabular}

Fig. 1. Structures of the compounds studied.

nitrogen shielding rather than nitrogen chemical shift. The two terms are equivalent in magnitude but are of opposite sign [2]. The convention we use bears a direct relationship to the nuclear magnetic shielding constant $\sigma$, a precisely defined physical quantity, and the results reported, considering the details reported in Section 2, are simply differences between the relevant shielding constants of the molecules studied and that of the reference employed.

\section{Experimental}

Compounds (1-9) were prepared according to a published procedure [3], with a modification where 2-fluoro-5-nitro-6-methylpyridine as a substrate was replaced with the corresponding chloro-derivative, and where the reactions were carried out in methanol rather than in ethanol as a solvent using the corresponding amine as a reagent. Compound (10) was obtained as in Ref. [4]; the synthesis of compounds $(11,12)$ was a modification of that in Ref. [5], with temperature lowered to $-10^{\circ} \mathrm{C}$ and $\mathrm{HNO}_{3}(d=1.4)$ as a nitrating agent.

The nitrogen magnetic shieldings (chemical shifts) of the systems studied were measured using high-precision ${ }^{14} \mathrm{~N}$ NMR spectra; these were taken on a Bruker AM-500 spectrometer $(11.7 \mathrm{~T})$ at $35.0 \pm 0.2^{\circ} \mathrm{C}$, as maintained by a VT unit, at a frequency of $36.14 \mathrm{MHz}\left(90^{\circ}\right.$ pulse, $40 \mu \mathrm{s}$; acquisition $0.111 \mathrm{~s} ; 4.521 \mathrm{~Hz} / \mathrm{pt}$ ). Efforts were made in order to reduce random and systematic errors in the 
shieldings to below $0.1 \mathrm{ppm}$. External liquid nitromethane was employed as a reference by means of $10 \mathrm{~mm} / 4 \mathrm{~mm}$ o.d. coaxial tubes, where the inner tube contained $0.3 \mathrm{M}$ nitromethane in acetone- $\mathrm{d}_{6}$ as a direct reference and a source of deuterium lock; the nitrogen shielding of this solution is $+0.77 \mathrm{pm}$ from that of neat liquid nitromethane [2]. This value is obtained from measurements using concentric spherical sample/reference containers in order to eliminate magnetic bulk susceptibility effects. The value of $+0.77 \mathrm{ppm}$ is used as a conversion constant, and since the measurements were taken for dilute solutions in pure acetone, the values of the shieldings thus obtained, with respect to that in neat liquid nitromethane, are bulk-susceptibility corrected. The exact resonance frequency of the ${ }^{14} \mathrm{~N}$ signal of neat nitromethane was $36.141524 \mathrm{MHz}$, from which a value of $36.136826 \mathrm{MHz}$ was obtained for the bare nitrogen nucleus [2]. The latter value was used in conjunction with the relevant resonance frequency differences to calculate the nitrogen shieldings relative to that of neat nitromethane. Lorentzian lineshape fitting of the ${ }^{14} \mathrm{~N}$ resonance signals was employed to produce values for the precise resonance frequencies of both the samples and the reference used. A very pure and dry acetone solvent was used as reported previously [6], and the solutions were prepared and handled under a dry argon atmosphere.

\section{Results and discussion}

The nitrogen NMR shieldings of $0.1 \mathrm{M}$ solutions of compounds (1-12) in acetone are reported in Table 1 together with that for unsubstituted pyridine under the same experimental conditions. The shielding of the 5-nitro nitrogen shows only little variation throughout the series, within less than $2 \mathrm{ppm}$, and does not seem to be significantly affected by substituents at the 2-amino moiety even if those include N-nitro or N-nitroso groups in addition to a variety of alkyl or aryl groups. In theory, one can expect some significant conjugation to take place between the $\pi$-electrons of the 5-nitro and 2-amino groups, as depicted in Fig. 2, provided that the molecular system is planar, and thus any

Table 1

Nitrogen NMR shieldings of the compounds studied referenced to neat liquid nitromethane, bulk-susceptibility corrected, $32^{\circ} \mathrm{C}$

\begin{tabular}{cccc}
\hline $\begin{array}{c}\text { Compound } \\
(0.1 \text { M solution } \\
\text { in acetone })\end{array}$ & \multicolumn{3}{c}{$\begin{array}{c}\text { Nitrogen NMR shielding } \\
(\mathrm{ppm})\end{array}$} \\
\cline { 2 - 4 } & pyridine nitrogen & nitro group & $\begin{array}{c}\text { amino substituent } \\
\text { nitrogen(s) }\end{array}$ \\
\hline $\mathbf{1}$ & +110.33 & +8.27 & +297.16 \\
$\mathbf{2}$ & +116.30 & +8.28 & +297.87 \\
$\mathbf{3}$ & +111.21 & +8.21 & +301.44 \\
$\mathbf{4}$ & +115.45 & +8.10 & +280.56 \\
$\mathbf{5}$ & +115.24 & +8.03 & +283.02 \\
$\mathbf{6}$ & +115.52 & +8.27 & +283.12 \\
$\mathbf{7}$ & +112.53 & +8.63 & +266.38 \\
$\mathbf{8}$ & +111.67 & +8.74 & +277.82 \\
$\mathbf{9}$ & +109.92 & +8.72 & +286.81 \\
$\mathbf{1 0}$ & +91.76 & +9.78 & $+115.87(\mathrm{NMe})$ \\
& & & $-183.25(\mathrm{~N}=\mathrm{O})$ \\
$\mathbf{1 1}$ & +78.53 & $+9.89(\mathrm{C}-\mathrm{nitro})$ & $+188.53(\mathrm{NMe})$ \\
& & & $+32.92(\mathrm{~N}-\mathrm{nitro})$ \\
$\mathbf{1 2}$ & +78.09 & $+9.84(\mathrm{C}-\mathrm{nitro})$ & $+176.67(\mathrm{NEt})$ \\
& & & $+34.61(\mathrm{~N}-\mathrm{nitro})$ \\
\hline
\end{tabular}


<smiles>[R]N(C)c1ccc([N+](=O)[O-])c(C)n1</smiles>

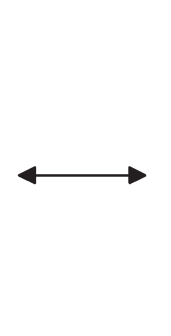<smiles>[R][N+]([R])=C1C=CC(=[N+]([O-])[O-])C(C)=N1</smiles>

Fig. 2. Potential conjugation effects between 2-amino and 5-nitro substituents of a pyridine ring which seem to be hampered by steric hindrance between 6-methyl and 5-nitro groups. The latter should force the nitro group out of the plane of the pyridine ring.

effects exerted by substituents $\mathrm{R}$ should be transmitted to the nitro group, affecting the electron charge distribution in the vicinity of the nitro nitrogen atom. However, the transmission should be quenched if the nitro group is forced out of the plane owing to the presence of 2-methyl substituent, and this seems to be the case in compounds (1-12), with the concomitant insensitivity of the nitro nitrogen shielding to long-range substituent effects. This point will be raised again later, in conjunction with semi-empirical quantum-mechanical calculations of the relevant atomic charges in the molecules concerned.

The shieldings of the nitrogen nuclei in the amino substituents involved seem to be typical of amino groups which bear at least one aryl substituent [2]. Here, the difference between compounds (2) and (4) as well as that between (11) and (12) represent the so-called $\beta$-effect of alkyl groups [2] which results in a significant deshielding of nitrogen in an $\mathrm{N}-\mathrm{R}$ moiety if one changes $\mathrm{R}$ within the sequence: methyl, ethyl, iso-propyl, tert-butyl, provided that there is no significant steric hindrance involved. In the present case, the latter seems to be responsible for the absence of the $\beta$-effect on the amino nitrogens in compounds (5) and (6), with respect to (2) and (4), since the substituted pyridine rings are likely to interact sterically with the corresponding iso-propyl and tert-butyl substituents of the amino groups. The phenylamino substituent in compound (7) shows a significant deshielding of its nitrogen nucleus with respect to the corresponding alkylamino substituents in (1-5), and this is also typical of arylamines [2], as a result of $\pi$-electron delocalization which includes the lone-pair electrons of the amino nitrogen atom; the same applies to the nitrogen shieldings of the amino moieties in the nitramino and nitrosoamino structures (10-12).

The most interesting, from the point of view of amino substituent effects on the electron charge distribution in pyridine rings, are the nitrogen shieldings of the pyridine nitrogen atom (Table 1). In the molecules examined, the 2-amino substituent exerts a remarkable shielding effect on the pyridine nitrogen with respect to the nitrogen NMR signal of pyridine itself. It amounts to about $+50 \mathrm{ppm}$ on the shielding scale, and is comparable to that observed for simple 2-aminopyridines [2]. Thus, the presence of 5-nitro and 6-methyl substituents in the compounds concerned does not seem to disturb significantly the pyridine nitrogen shielding of the parent 2-aminopyridine structure. The origin of the shielding effect exerted by the 2-amino substituent can be intuitively assigned to $\pi$-electron delocalization where the lone-pair electrons of the amino nitrogen are involved (Fig. 3), producing an excess of negative charge at the pyridine nitrogen atom. In order to verify this we carried out quantummechanical calculations at the level of the ZINDO/1 method [7] (Zerner's INDO, an improved version of INDO) for some of the molecules studied, using molecular geometries which were optimized using the same method. The results are shown in Table 2, and they indicate that the 2-amino substituent in compound (2) produces a considerable excess of negative charge at the pyridine nitrogen as compared with parent pyridine. Concurrently, the optimized geometries show that the 5-nitro group is twisted, 
<smiles>[R]N([R])c1ccc([N+]([R])=O)c(C)n1</smiles>

Fig. 3. Potential conjugation effects between the 2-amino substituent and the pyridine nitrogen atom.

Table 2

Atomic charges at nitrogen calculated by the ZINDO/1 method

\begin{tabular}{lccc}
\hline Nitrogen atom & Pyridine & Compound 2 & Compound 11 \\
\hline in pyridine ring & -0.203 & -0.311 & -0.250 \\
in nitro group & - & +0.569 & +0.567 \\
in amino group & - & -0.234 & -0.137 \\
in N-nitro group & - & - & +0.654 \\
\hline
\end{tabular}

by about $20^{\circ}$, from the plane of the pyridine ring, in accord with the foregoing discussion of the nitrogen shieldings of the nitro groups. We have also carried out geometry optimization for analogs of compounds (1) and (3), those devoid of the 6-methyl substituent, and the calculations indicate that in this case the nitro group is coplanar with the aromatic ring.

Alkyl and aryl substituents attached to the 2-amino groups exert rather modest effects on the pyridine nitrogen shielding (Table 1) in the structures examined, but the corresponding N-nitroso and N-nitro substituents induce large deshielding shifts, by about 20 and $30 \mathrm{ppm}$, respectively. However, the pyridine nitrogen shieldings in these derivatives are still higher than that in pyridine itself, and this suggests that the effect of the 2-amino substituent in such 2-nitrosoamino and 2-nitramino derivatives is quenched, but not completely, and that there should still be an excess of negative charge at the pyridine nitrogen with respect to unsubstituted pyridine. All of this is borne out by the results of calculations presented in Table 2. The calculated atomic net charges for pyridine, -0.203 , for the 2-nitramino derivative (11),-0.250 , and for the 2-methylamino derivative (2), -0.311 , follow the direction of the increasing shielding of their pyridine nitrogens, $+62.5,+78.5$, and $+116.3 \mathrm{ppm}$, respectively. Thus, both the experimental nitrogen shieldings and the calculations suggest that the 2 -amino substituent induces an increase in electron charge density at the pyridine ring nitrogen even if the amino moiety bears electron sinks such as nitro or nitroso groups.

The calculations (Table 2) seem also to explain the lack of significant effects of substituents on the nitrogen shielding of the 5-nitro group. The net charges computed for the relevant nitrogen atoms in compounds (2) and (11) do not show any appreciable difference, in spite of the fact that in (11) a nitro group replaces the hydrogen at the 2 -amino moiety in (2).

In conclusion, the nitrogen shieldings of pyridine systems seem to provide an attractive tool for monitoring electron charge redistributions induced by substituents in such heteroaromatic stuctures, including $\pi$-electron conjugation and steric effects.

\section{References}

[1] G.V. Kulkarni and A. Ray, J. Mol. Struct. 71 (1981), 253.

[2] M. Witanowski, L. Stefaniak and G.A. Webb, Nitrogen NMR spectroscopy, in: Annual Reports on NMR Spectroscopy, Vol. 25, Academic Press, 1993 (and references therein). 
[3] Z. Talik and B. Brekiesz-Lewandowska, Roczniki Chem. 44 (1970), 1325.

[4] H. Ban-Oganowska, A. Puszko, B. Palasek and M. Wandas, Chem. Heterocycl. Comp. (in press).

[5] T. Talik and Z. Talik, Roczniki Chem. 41 (1967), 483.

[6] M. Witanowski, Z. Biedrzycka and G.A. Webb, Magn. Reson. Chem. 34 (1996), 233.

[7] The implementation of ZINDO/1 used was that included in ${ }^{\mathrm{TM}}$ HyperChem-4 software suite (Hypercube, Inc.). 


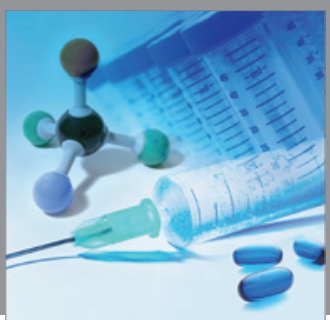

International Journal of

Medicinal Chemistry

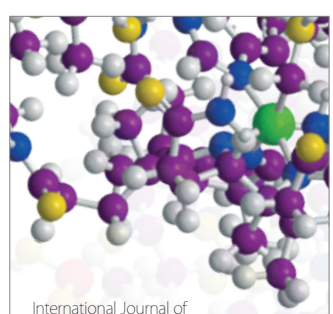

Carbohydrate Chemistry

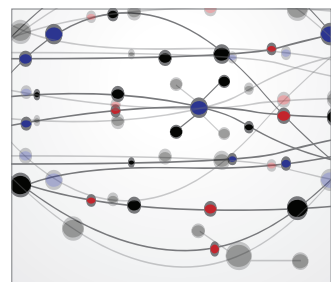

The Scientific World Journal
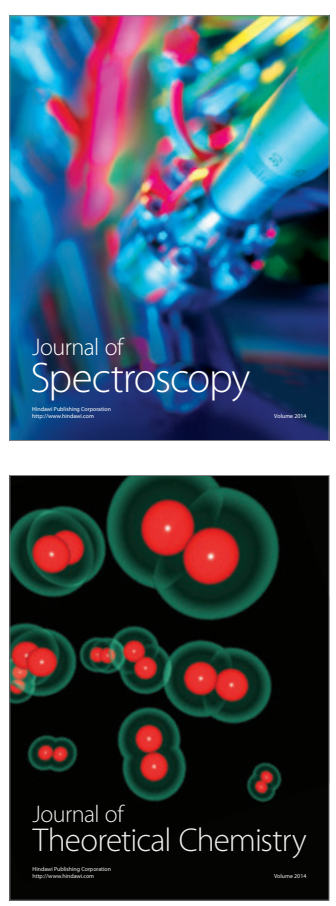
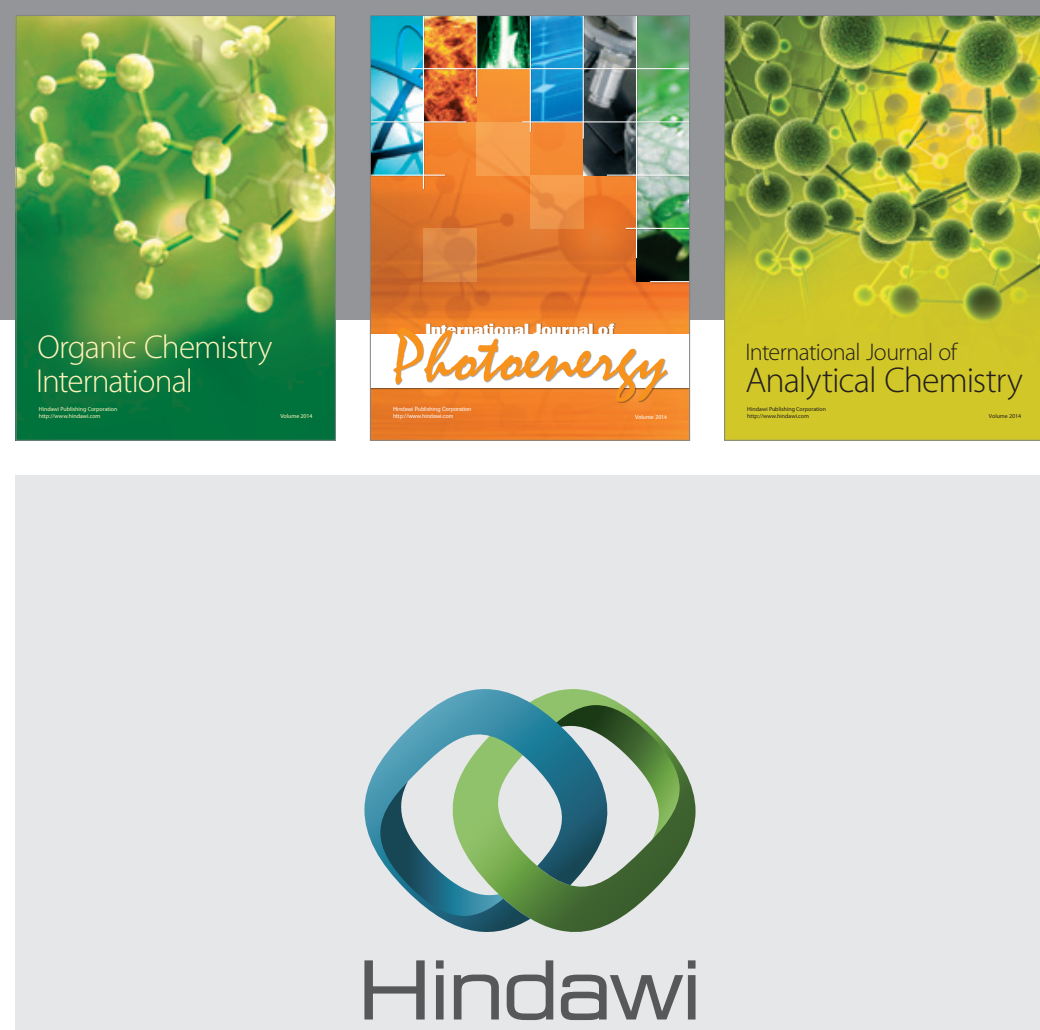

Submit your manuscripts at

http://www.hindawi.com
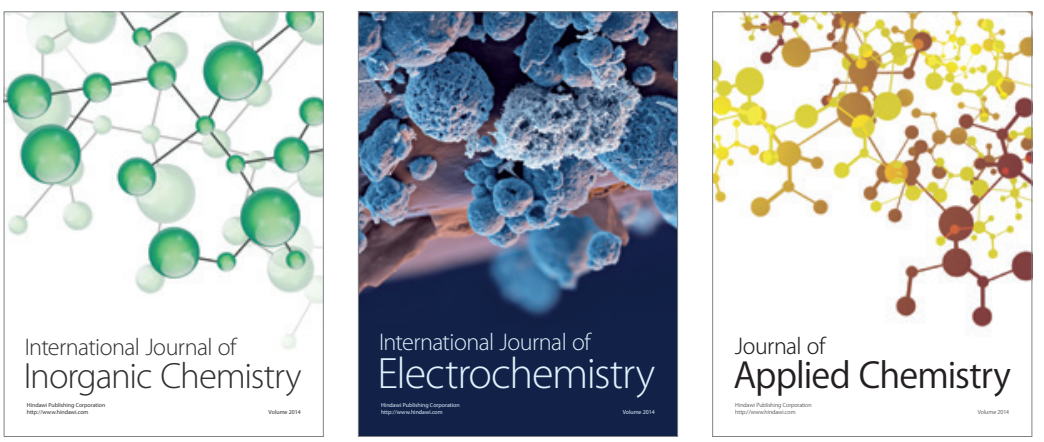

Journal of

Applied Chemistry
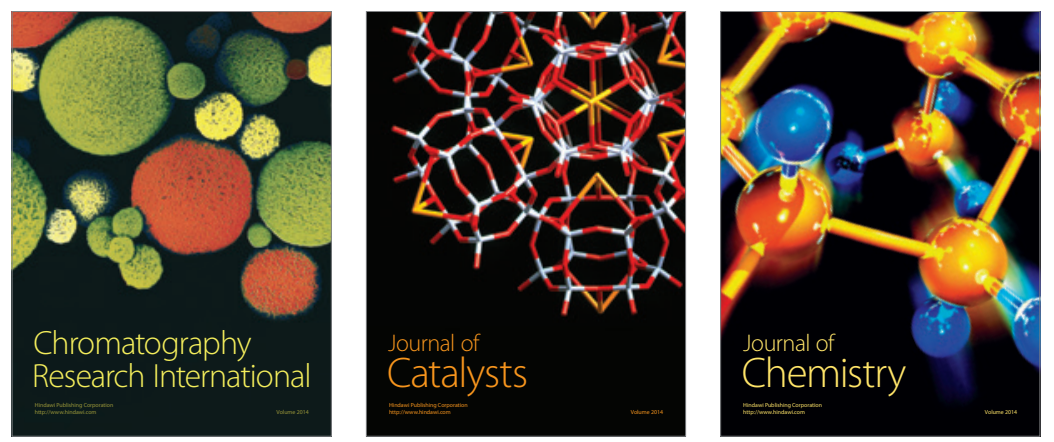
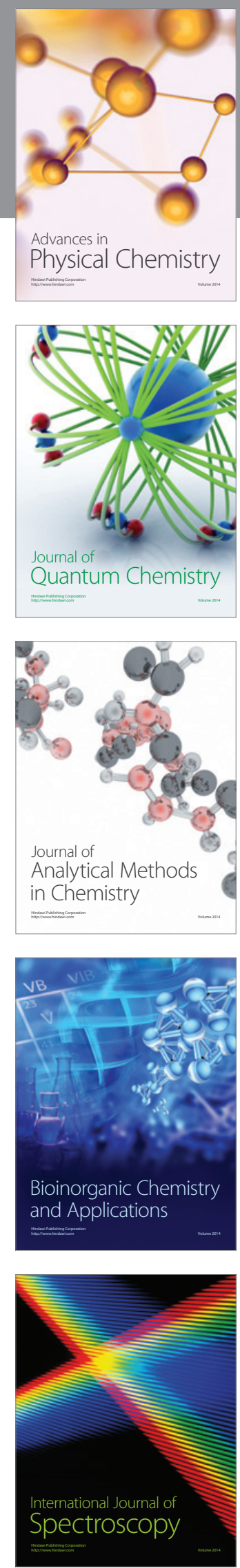